\title{
PJI with Sepsis, a Complex Emergency Situation with Prodromal Stage
}

\author{
Nils Haustedt ${ }^{1}$, Jorge Armando González Chapa ${ }^{2}$, Moritz Hentschke ${ }^{3}$ \\ ${ }^{1}$ Clinic of Septic Bone and Soft-Tissue Surgery, Schön-Klinik Hamburg Eilbek, Hamburg, Germany \\ ${ }^{2}$ Departamento de Inmunología, Facultad de Medicina, UANL, Monterrey, Mexico \\ ${ }^{3}$ Departement of Microbiology and Virology, Labor Fenner, Hamburg, Germany \\ Email: nhaustedt@schoen-klinik.de, jorgechapag89@gmail.com,mhentschke@fennerlabor.de
}

How to cite this paper: Haustedt, N., Chapa, J.A.G. and Hentschke, M. (2020) PJI with Sepsis, a Complex Emergency Situation with Prodromal Stage. Open Journal of Orthopedics, 10, 203-220.

https://doi.org/10.4236/ojo.2020.109023

Received: July 30, 2020

Accepted: September 8, 2020

Published: September 11, 2020

Copyright (c) 2020 by author(s) and Scientific Research Publishing Inc. This work is licensed under the Creative Commons Attribution International License (CC BY 4.0).

http://creativecommons.org/licenses/by/4.0/

\section{(c) (i) Open Access}

\begin{abstract}
Introduction: With a considerable morbidity, mortality and a high financial impact, PJI has been considered as one of the most serious complications after arthroplasty. Special awareness must be given to these patients due to a life threatening systemic PJI related complication, sepsis. A prompt detection and adequate medical management of this situation is crucial for avoiding unfavorable outcomes. Methods: Between January 2011 and December 2012, we retrospectively examined adult patients who met PJI-SIRS criteria. Medical history search for patients' anamnesis, surgical times, laboratory-microbiological findings and success rates was performed. Results: Twenty patients were enrolled in this study with a mean age of 71.35 years. Men population was more commonly affected with $55 \%$ of the cases. The knee was mainly the affected joint with $52 \%$. A severe systemic disease was present in $80 \%$ of the cases. A sickness sensation and pain were the most common prodromal symptoms found 4.9 days before the admission to the hospital. In $85.7 \%$ of the cases the etiological agent was identified, with Staphylococcus aureus as the most common. Two-stage surgery treatment was performed with a time interval between admission and the first operation of $5.45 \mathrm{~h}$. The mean time during the first and second operation was of 23 minutes and 117 minutes respectively. A reduction on microbiological positive cultures was found after the first rapid operation $(P=0.0038)$. Serum CRP levels and fever disappearance were the first parameters which indicated a favorable disease evolution after the first operation $(P=0.0137)$ and $(P=0.0181)$ respectively. A successful management was possible in $90 \%$ of the cases. Conclusion: Sepsis in patients with PJI announces itself early through the leading symptoms of feeling sick and pain in the affected joint. This time interval should be used therapeutically and diagnostically to prevent the complication of a life-threatening sepsis at best.
\end{abstract}




\section{Keywords}

Arthroplasty, Sepsis, PJI, Septic Arthritis, SIRS

\section{Introduction}

With higher life expectancy and new therapeutic approaches for joint diseases, the general population has increasingly higher expectations of mobility in old age. This leads to a steady increase in the number of joint replacement operations, which is expected to rise by $137 \%$ and $601 \%$ for total hip and knee endoprostheses by 2030 [1]. Although endoprostheses are successful surgical procedures, with more than $95 \%$ of survivors undergoing surgery within a follow-up period of 10 years [1], the number of periprosthetic joint infections (PJI) will also increase. PJI is a devastating and serious complication after arthroplasty. PJI is considered a rare event that occurs in about $2 \%$ of primary and $4 \%$ of revision endoprostheses [2] [3] and its incidence is lower than that of aseptic loosening [4]. Nevertheless, it can lead to more serious complications for endoprostheses, such as prolonged hospitalization, multiple operations, functional impairment and even death, for which a five-year mortality rate of $21.12 \%$ for total hip endoprostheses and $14.4 \%$ for total knee endoprostheses after 3.8 years has been reported [5] [6].

There are different classification schemes for PJI. The classification into acute and chronic PJI is the most frequently used and published [7]. The most important criterion of these classifications is the condition and formation of a bacterial biofilm. It is assumed that acute PJI begins $<4$ weeks after prosthesis implantation or, in the case of hematogenic etiology, has a maximum symptom duration of less than 3 weeks. In these cases, the biofilm is not yet fully developed. These acute PJIs are most frequently triggered by relatively highly virulent microorganisms such as Staphylococcus aureus and Gram-negative bacteria. In contrast, chronic PJI (low grade) are delayed postoperative infections, i.e. >than 4 weeks after prosthesis implantation or, in the case of hematogenic genesis, a symptom duration of more than 3 weeks [7] [8]. The biofilm age of this type of infection is usually mature. The main germs of chronic PJI are relatively low-virulent microorganisms, such as coagulase-negative staphylococci or propionibacterium acnes [7] [8].

The most life-threatening complication for patients with PJI is the sepsis-disease. Patients with PJI may develop bacteremia, which can trigger a systemic inflammatory response (SIRS) through certain bacterial components such as endotoxins recognized by immune cells [9]. The combination of infection and SIRS is called sepsis. In the sepsis spectrum, SIRS is the first complication step, which also includes severe sepsis and septic shock with organ dysfunction [10]. It has been described that mortality in patients with severe sepsis and septic shock is about 50\%, which is almost 6 times higher than in patients without sepsis [11] 
[12].

Early detection and rapid adequate treatment of the early stage of sepsis in patients with PJI is crucial for the prognosis and prevention of higher mortality.

\section{Methods}

\subsection{Study Group}

We retrospectively collected data from 20 adult patients diagnosed with PJI who met the SIRS criteria [13], who were admitted and treated at our clinic between January 2011 and December 2012. This represented 7.5\% of all 286 patients with PJI at our clinic during this period. SIRS was defined according to the criteria of the German Sepsis Society. In short: fever or hypothermia, tachycardia, tachypnea, leukocytosis or leukopenia were used as criteria parameters. In the presence of a positive blood culture and 2 of these parameters, the patients were defined as patients with SIRS. In the presence of a negative blood culture, patients must have 4 of these parameters to define a SIRS. Exclusion criteria consisted in patients under 18 years old and an incomplete medical record. The retrospectively evaluated data of our study was anonymized and the information was only accessible to the treatment team.

\subsection{Therapy Concept}

The treatment concept for the patients included emergency diagnostics and a two-stage operation concept. The diagnosis included a complete anamnesis with recording the type and onset of the prodromal symptoms such as joint redness, heat, swelling, pain, fever and feeling of illness. The two-stage surgical concept included an initial immediate emergency operation with arthrotomy of the joint, synovectomy and debridement. This surgery also included joint lavage, the use of antibiotic labels and the placement of a drainage tube for subsequent daily sterile joint lavage, until a second delayed and planned surgical revision. At this time, the microbiological culture results of the primary diagnosis and primary surgery had to be available. The second surgery included debridement, joint lavage and the use of absorbable antibiotic sponges. Only in case of prosthesis loosening or the microbiological findings of a multi-resistant pathogen we saw the indication for prosthesis explantation.

\subsection{Samples and Laboratory Tests}

In the diagnostic phase a preoperative joint puncture was performed for synovial fluid samples. A synovial fluid WBC count and a microbiological culture were performed. This phase also included radiographs to identify prosthesis loosening and the type of prosthesis. Peripheral blood samples were taken to determine WBC count, inflammation markers such as CRP, coagulation tests, kidney and liver function parameters, electrolytes and procalcitonin levels. In addition, blood cultures for aerobic and anaerobic microorganisms were prepared at patient admission. C-reactive protein and WBC count in peripheral blood were 
measured daily throughout the patient's hospital stay. Microbiological cultures were also established from synovial fluid/tissue samples collected during the first and second surgery.

\subsection{ASA Classification}

In addition, the classification of the physical status of the American Society of Anesthesiologists (ASA) [14], which was performed by a specialist on the day of the patient's anesthesia treatment, was added to our database. In short, ASA 1 is considered a healthy person, ASA 2 is a patient with a mild systemic disease, ASA 3 is a patient with a severe systemic disease, ASA 4 is a severe systemic disease that poses a constant threat to the patient's life, ASA 5 is a moribund person who is not expected to survive without surgery, and an ASA 6 classification, i.e. a person declared brain dead whose organs have been removed for donor purposes.

\subsection{Statistical Analyses}

All the data was analyzed using descriptive statistics, all patients narratives and clinical information during the anamneses, diagnosis and treatment was converted into structured data). The Mann Whitney non-parametric Gaussian distribution test was used between 2 groups of data. Fisher exact test was used to analyze microbiological culture data using Prism GraphPad 8 (San Diego, California).

\section{Results}

\section{Demographic and clinical characteristics of the patients}

As shown in Table 1 a total of twenty patients were enrolled in this study with a follow up time mean of 9.4 months (range from 3 - 27 months). The mean age of the patients was of 71.35 years old with a range from $45-89$ years. Men were more commonly affected with a $55 \%$ of the cases (11 patients) compared to women with a $45 \%$ (9 patients). A slight difference was found between ages of men and women which had a mean of 69.6 years $(49$ - 82) and 73.6 years (45 89) respectively. The most common affected joint was the knee with a $52 \%$ of the cases (10 patients,), followed by hip with a $43 \%$ and shoulder $5 \%$. The vast majority of the patients presented a mono articular PJI (95\%) and only 1 patient had the affection of the 2 knee joints. The time interval between the prosthesis implantation and the PJI was of 57.6 months (5 - 264). The inpatient length stay had a mean of 21 days (14 - 36 days).

\section{Most of the patients had a severe systemic disease ASA classification}

As shown in Figure 1 none of the patients was classified as a healthy ASA 1 patient. The highest number of patients $80 \%$ (16 patients) were classified as patients with severe systemic disease, ASA 3 classification. On the other hand, ASA 2 had only $10 \%$ of the patients, alike ASA 4 classification. No ASA 5 or 6 classified patients were enrolled. 


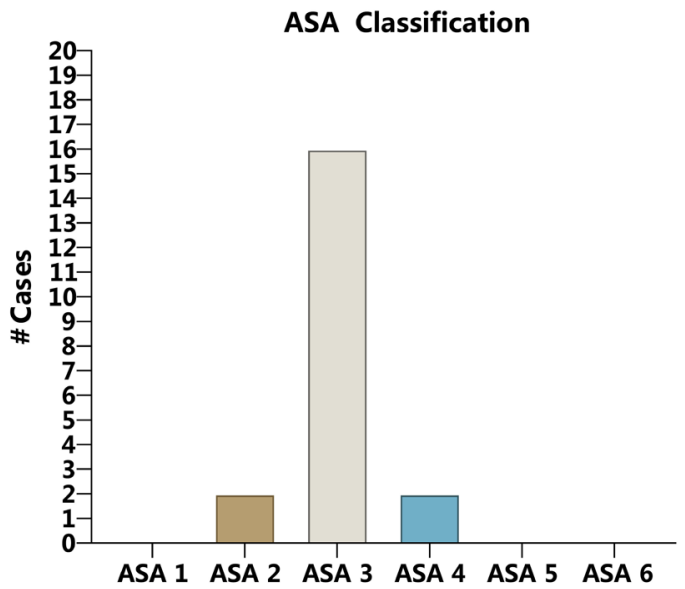

Figure 1. Most of the patients had a severe systemic disease ASA classification.

Table 1. Demographic and clinical characteristics of the patients.

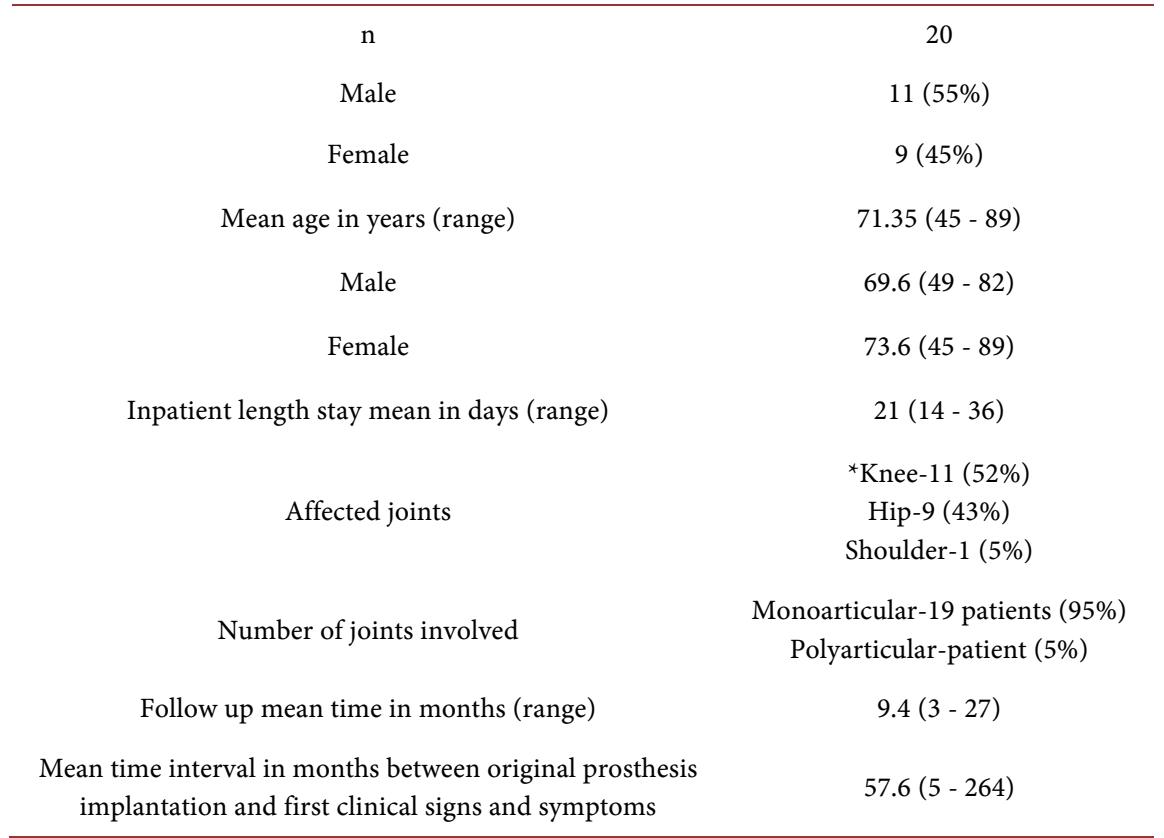

${ }^{*} 1$ patient with 2 knee PJI.

\section{A two-step operation treatment focused on a rapid first operation}

The time interval between the admission and the first operation was of $5.45 \mathrm{~h}$ $(2.5-12 \mathrm{~h})$. On the other hand, the time gap between the first and second operation was of 8 days ( $4-12$ days). The mean duration of the first and second operation was of 23 minutes (13 - 52) and 115.7 minutes (71 - 208) respectively.

In those patients were conservation of the prosthesis was opted a mean duration of operation of 83.3 minutes was observed, in contrast to those where the prosthesis needed to be removed which lasted a mean duration of 148 minutes. The time gap between the removal and replacement of the prosthesis had a mean of 6.3 weeks (Figure 2).

A 4.9 days window for identifying main prodromes. 


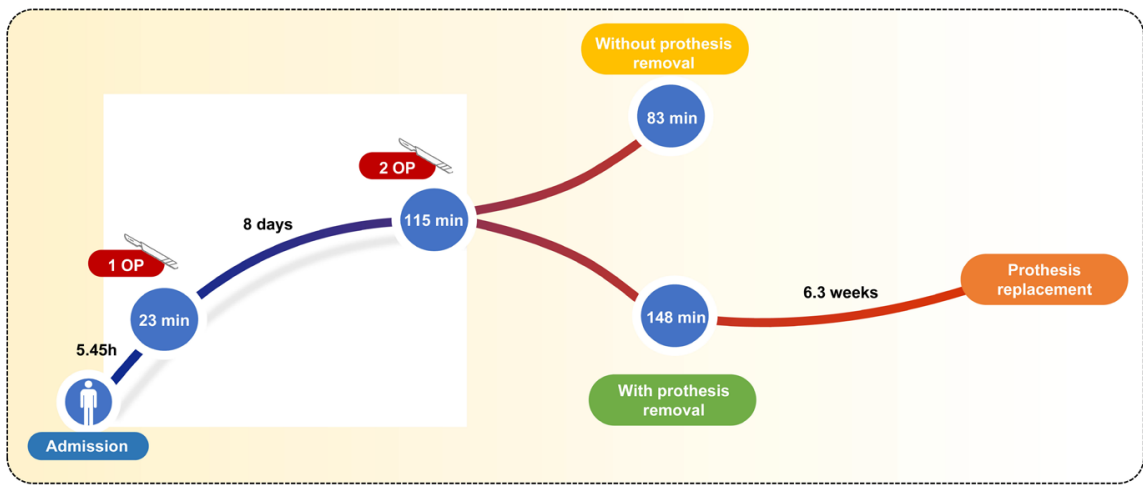

Figure 2. A two-step operation treatment focused on a rapid first operation.

Probable prodromal signs or symptoms were presented by $90 \%$ of the patients before admission, leaving $10 \%$ without any prodromal variable to measure (Table 2). A sickness sensation and pain were the most common prodromal symptoms patients presented with a $90 \%$ and $85 \%$ respectively. These was followed by joint swelling in a $70 \%$ of the cases. Fever and joint warmth were present in $60 \%$ while the less common prodrome was the joint redness with a $25 \%$. The time interval of onset of illness before inpatient treatment and the appearance of these prodromal variables was of 4.9 days ( 1 - 10 days).

Microbiological cultures \% of positiveness decreases 3 times after the first rapid operation

In the $85.7 \%$ of the cases (17 patients) the isolation and identification of the etiological agent was possible during the preoperative joint puncture samples. On the other hand, negative cultures were present in a $14.3 \%$ ( 3 patients), which had systemic antibiotic systemic treatment before the sampling (Figure 3 ).

The most common isolated bacteria in synovial fluid samples belonged to Staphylococcus spp. and Streptococcus spp. with a 52.2\% and 28.6\% respectively, leaving another $4.8 \%$ for other bacteria species. Staphylococcus aureus was responsible for more than the half of the cases of the Staphylococcus spp followed by Staphylococcus epidermidis. On the other hand, Streptococcus pneumoniae was the most common Streptococcus spp isolated in this group (Table 3).

Figure 3 demonstrates that microbiological cultures made from the first intra operation soft tissue samples showed a $75 \%$ of positiveness while $25 \%$ presented a negative culture. This percentages were totally inverted after this first fast surgery treatment with negative and positive cultures of $75 \%$ and $25 \%$ respectively ( $P=0.0038)$ at the moment of the second operation sampling. On the other hand, blood cultures were mainly negative with a $75 \%$ while in the $25 \%$ of the cases presented a positive culture. All microbiological findings are summarized in Table 4.

\section{CRP serum levels decreased after first rapid operation}

Serum C-reactive protein showed statistically significant lower levels the next day after the first rapid surgery $(P=0.0137)$ as shown in Figure 4. A range from $87-337 \mathrm{mg} / \mathrm{L}$ with a median and mean values of $267 \mathrm{mg} / \mathrm{L}$ and $247 \mathrm{mg} / \mathrm{L}$ 


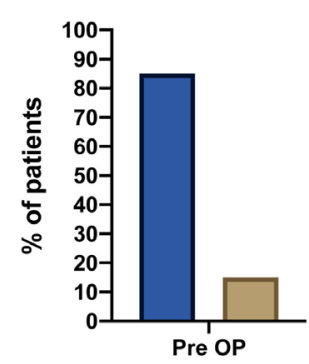

(a)

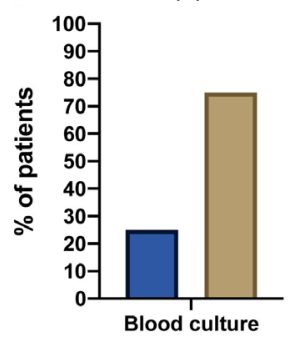

(c)

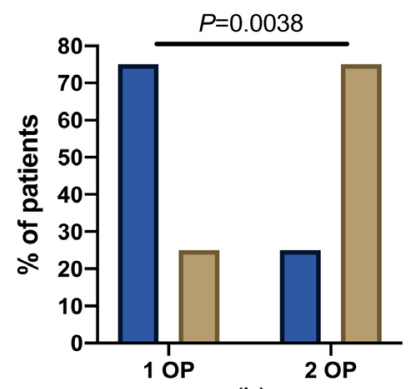

(b)

positive culture

negative culture

Figure 3. Microbiological cultures \% of positiveness. Positive cultures decrease 3 times after the first rapid operation. (a) Descriptive statistics in \% during preoperative cultures (joint puncture); (b) Fisher's exact test between 1OP and 2OP; (c) Descriptive statistics in $\%$ during preoperative blood cultures.

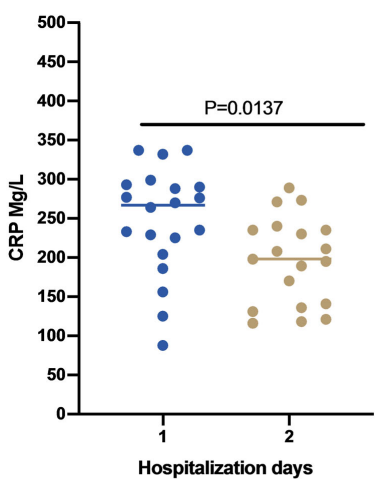

(a)

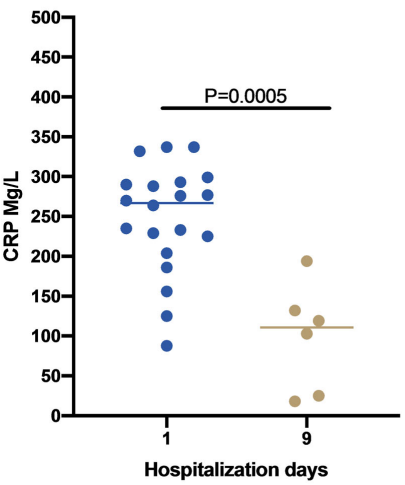

(c)

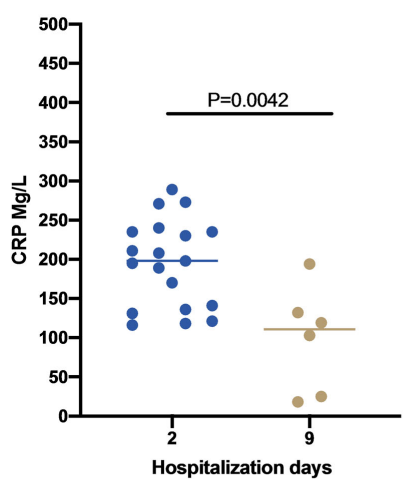

(b)

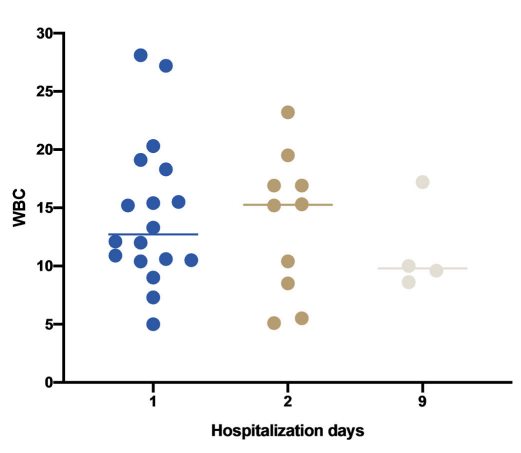

(d)

Figure 4. CRP serum concentration through the patient in care management. CRP levels decreased after first rapid operation at day 2 and after the second operation at day 9. Mann Whitney test. (a) Day $1 \mathrm{n}=20$, day $2 \mathrm{n}=19$; (b) day $2 \mathrm{n}=19$, day $9 \mathrm{n}=6$ (d) WBC count per microliter day $1 \mathrm{n}=18$, day $2 \mathrm{n}=10$ and day $3 \mathrm{n}=4$. 
Table 2. Prodrome signs and symptoms in patients with PJI.

\begin{tabular}{cc}
\hline \multicolumn{2}{c}{ Prodromal signs and symptoms } \\
\hline Sign and sympotm & $\%$ of appearance \\
\hline Sickness sensation & $90 \%$ \\
Pain & $85 \%$ \\
Joint swelling & $70 \%$ \\
Fever & $60 \%$ \\
Joint warmth & $60 \%$ \\
Joint redness & $25 \%$
\end{tabular}

Table 3. Etiological agents found in joint samples.

\begin{tabular}{cc}
\hline \multicolumn{2}{c}{ Preoperative microbiological cultures } \\
\hline Bacterium spp & $\%$ of cases \\
\hline Staphylococcus & $52.4 \%(11)$ \\
Staphylococcus aureus & $28.6 \%(6)$ \\
\hline MRSA “Methicillin resistant Staphylococcus aureus" & $4.8 \%(1)$ \\
Staphylococcus epidermidis & $9.5 \%(2)$ \\
Staphylococcus lugdunensis & $4.8 \%(1)$ \\
Staphylococcus warneri & $4.8 \%(1)$ \\
Streptococcus & $28.6 \%(6)$ \\
Streptococcus pneumoniae & $9.5 \%(2)$ \\
Streptococcus oralis & $4.7 \%(1)$ \\
Streptococcus harei & $4.7 \%(1)$ \\
Beta hemolytic streptococcus & $4.7 \%(1)$ \\
Streptococcus mutans & $4.7 \%(1$ \\
Viridans streptococci & $4.7 \%(1)$ \\
Others & $4.8 \%$ \\
\hline
\end{tabular}

Table 4. Microbiological cultures results.

\begin{tabular}{cccc}
\hline $\begin{array}{c}\text { Pre OP } \\
\text { Blood sample }\end{array}$ & $\begin{array}{c}\text { Pre Op } \\
\text { joint punkture }\end{array}$ & $\begin{array}{c}\text { First OP } \\
\text { joint sample }\end{array}$ & $\begin{array}{c}\text { Second OP } \\
\text { joint sample }\end{array}$ \\
\hline & Streptococcus oralis & Streptococcus oralis & \\
& Peptoniphilus harei & $\begin{array}{c}\text { Proteus mirabilis, Group B } \\
\text { beta hemolytic streptococci }\end{array}$ & \\
& Staphylococcus & Staphylococcus & Staphylococcus \\
lugdunensis & lugdunensis & \\
Staphylococcus aureus & Staphylococcus aureus & \\
multisensible & multisensible & \\
Staphylococcus & Staphylococcus & \\
aureus susceptible & aureus susceptible & \\
Streptococcus viridans & Streptococcus viridans & \\
\hline
\end{tabular}




\begin{tabular}{|c|c|c|c|}
\hline Continued & & & \\
\hline \multirow[t]{5}{*}{$\begin{array}{c}\text { Staphylococcus } \\
\text { aureus }\end{array}$} & $\begin{array}{l}\text { Staphylococcus aureus } \\
\text { multisensible }\end{array}$ & Staphylococcus aureus & \\
\hline & $\begin{array}{l}\text { Group B beta } \\
\text { hemolytic streptococci }\end{array}$ & $\begin{array}{c}\text { Group B beta hemolytic } \\
\text { streptococci }\end{array}$ & $\begin{array}{c}\text { Staphylococcus } \\
\text { epidermidis }\end{array}$ \\
\hline & $\begin{array}{l}\text { Staphylococcus } \\
\text { epidermidis }\end{array}$ & Staphylococcus eidermidis & $\begin{array}{c}\text { Staphylococcus } \\
\text { epidermidis }\end{array}$ \\
\hline & $\begin{array}{l}\text { Staphylococcus } \\
\text { epidermidis }\end{array}$ & & $\begin{array}{c}\text { Staphylococcus } \\
\text { epidermidis }\end{array}$ \\
\hline & Staphylococcus aureus & Staphylococcus aureus & \\
\hline MRSA & MRSA & MRSA & \\
\hline $\begin{array}{l}\text { Streptococcus } \\
\text { penumoneae }\end{array}$ & $\begin{array}{l}\text { Steptococcus } \\
\text { pneumonieae }\end{array}$ & Streptococcus pneumoniea & \\
\hline \multirow[t]{2}{*}{$\begin{array}{c}\text { Staphylococcus } \\
\text { aureus }\end{array}$} & Staphylococcus aureus & Staphylococcus aureus & $\begin{array}{c}\text { Staphylococcus } \\
\text { aureus }\end{array}$ \\
\hline & Streptococcus mutans & Streptococcus mutans & \\
\hline \multirow[t]{2}{*}{$\begin{array}{l}\text { Steptococcus } \\
\text { pneumonieae }\end{array}$} & Steptococcus pneumonieae & Steptococcus pneumonieae & \\
\hline & Staphylococcus warneri & & \\
\hline
\end{tabular}

respectively were observed during the first day of hospitalization. The next day after the first surgery the range was from $116-289 \mathrm{mg} / \mathrm{L}$ with a median value of $198 \mathrm{mg} / \mathrm{L}$ and a mean of $195 \mathrm{mg} / \mathrm{L}$.

After the second surgery these CRP serum levels decreased almost 2 times $(P$ $=0.0042)$ with a range from 18.10 to $194 \mathrm{mg} / \mathrm{L}$ and a median and mean values of $98.52 \mathrm{mg} / \mathrm{L}$ and $111 \mathrm{mg} / \mathrm{L}$ respectively and almost 3 times when compared to the admission day levels $(P=0.0005)$. Peripheral blood WBC count didn't show a statistically significant difference through the days of the patient's hospitalization time $(P>0.05)$. Figure 5 shows the decrease tendency of CRP concentrations through patients medical management.

\section{Normal body temperature after second day of hospitalization}

During the admission, patients presented a body temperature mean of $38.60^{\circ} \mathrm{C}$ which decreased after the first rapid surgery to $37.32^{\circ} \mathrm{C}(P=0.0181)$ and $36.76^{\circ} \mathrm{C}(P=0.0006)$ on the second and third day of hospitalization respectively. From day 3 on patients body temperature remained without any statistically significant change (Figure 6).

Half of the patients had an infection resolution without prostheses removal

Our results showed that $100 \%$ of the patients belonging to the group treated without a protheses removal (10 patients) had a successful management of the infection with a follow up mean time of 10.8 months (range 5 - 27). On the other hand, patients whom underwent to a 2 -stage prosthesis exchange (8 patients) had a also successful treatment rate of $100 \%$ in controlling the infection with a follow up mean time of 7.8 months (range 3 - 18). One patient (5\%) died due to multiorgan disfunction syndrome related to multiple PJIs and a Girdlestone resection arthroplasty was needed also in $5 \%$ of the cases (Table 5). 


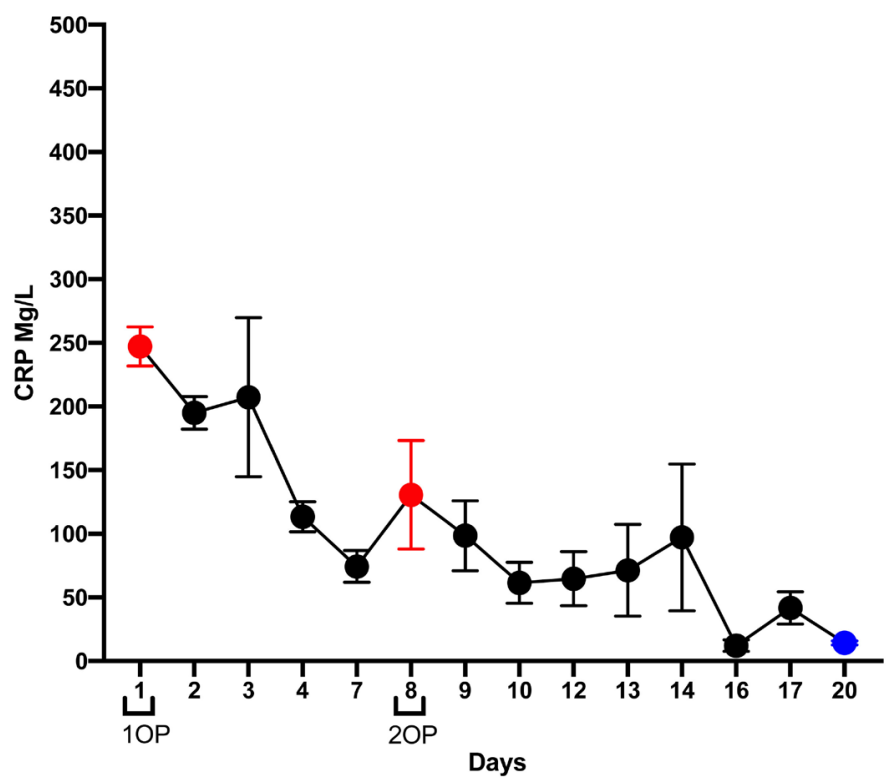

Figure 5. CRP serum concentration during the inpatient care days. CRP levels show decreased levels at day 2 after the first operation and after the second operation at day 8 . The lowest levels were at the final day of the patients in care treatment. Values are represented as means with SEM, a minimum of three samples per day from three different patients were considering for the graphic.

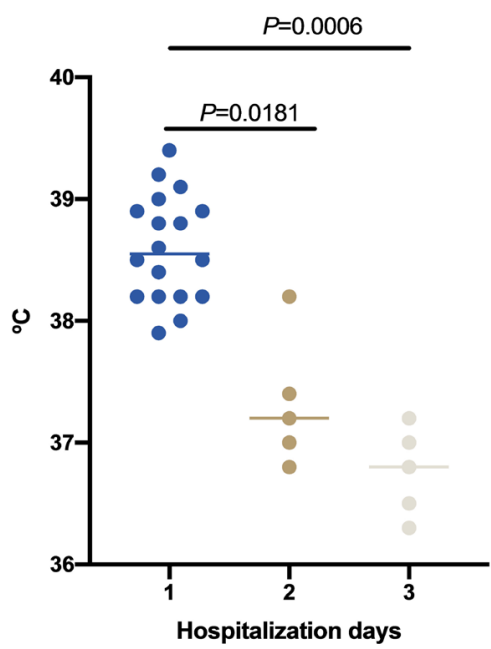

Figure 6. Patients body temperature during hospitalization days. Dunn's multiple comparison test day $1 \mathrm{n}=18$, day $2 \mathrm{n}=5$, day $3 \mathrm{n}=5$.

Table 5. PJI treatment surgery options outcome.

\begin{tabular}{ccc}
\hline & PJI surgeries & \\
\hline & $\%$ of cases (patients) & Successful rate \\
\hline Without prostheses removal & $50 \%(10)$ & $100 \%$ \\
With protheses removal & $40 \%(8)$ & $100 \%$ \\
Girdlestone situation & $5 \%(1)$ & - \\
Deaths & $5 \%(1)$ & -
\end{tabular}




\section{Discussion}

Although PJI occurs in less than 5\% of all endoprostheses [2] [3], it remains a severe, devastating and serious complication. PJI with sepsis in particular can also have systemic effects with increased mortality [15]. For this reason, the early and correct detection of early signs, symptoms and laboratory parameters is of crucial importance, not only to detect the joint infection early, but also to prevent higher mortality due to sepsis.

In our retrospective study we were able to show that on average 4.9 days before sepsis the first symptoms already appeared. Leading among the symptoms were joint pain and an unspecific feeling of illness. Our results showed that joint pain occurred in $85 \%$ of patients, which is consistent with other studies that showed its occurrence in almost $80 \%$ - 100\% of cases [16] [17] [18].

However, different from these studies, pain was not the most common manifestation the patients were presenting in our research. Most of our patients (90\%) described a general sickness sensation as the most common clinical manifestation before their arrival to the hospital. We were able to demonstrate that sepsis is preceded by a prodromal stage that should be used therapeutically and diagnostically.

Consistent with other studies the majority of our patients (55\%) were men, which population has been considered as a non-modifiable risk factor for developing PJI [19] [20] [21]. Although no scientific explanation has yet been proposed for this phenomenon a reasonable explanation could be job or comorbidities related which makes this population more susceptible for the infection. Our results showed a slight not significant difference in the age of the patient's affection, which was older in women (73.6 years) compared to men $(69.3$ years). One must take into consideration that although the importance of age in many other diseases, age alone has not been considered as risk factor for developing PJI [22].

Of the 3 different joints affected, the knee was most frequently affected with $52 \%$ of cases, followed by the hip with $42 \%$ and the shoulder with $5 \%$. This is consistent with previous studies that showed PJI rates between $0.5 \%-2 \%$ for all knee joint prostheses, followed by almost $1 \%$ for all hip and shoulder prostheses in less than $1 \%$ [23] [24].

Probably due to the fact that the knee is the largest joint in the human body, it is also the most commonly affected joint in PJI, which is also due to the greater mobility, larger prosthetic surface area and less soft tissue coverage. However, there could also be a bacterial cause. Certain bacteria, such as Staphylococcus aureus, have surface components that bind or attach to extracellular matrices, such as fibrinogen, collagen and elastin [25] [26]. These components, which are considered determinants of virulence [27], may also facilitate the attachment of the bacteria to implanted materials that are coated with host proteins after insertion [28]. This attachment to a foreign implanted material could develop a microbial community embedded in a protective extracellular matrix called biofilm 
[29].

As our results show, a positive blood culture could only be obtained in $25 \%$ of cases. Although bacteremia is a known consequence of PJI, these cultures are not often obtained or considered as part of the diagnostic criteria of PJI [30] [31]. It should be noted that if positive, they may reduce the success rate of PJI treatment [32] and even lead to death, as one of our patients who presented a positive MRSA blood culture and unfortunately died in septic shock. However, the role of blood cultures in PJI diagnosis is an issue that requires further research.

It is well known that Gram-positive bacteria are the most common bacteria involved in PJI. Staphylococcus aureus represents the most common etiological agent with more than the half of the cases [8]. This is accordingly to our results were this etiological agent represented the $52.2 \%$. However other study showed Staphylococcus epidermidis as the most common Gramm-positive cause of the infection [33] which in our case was the second most common. Our data showed that Streptococcus spp were present in a $28.6 \%$, which has been described to represent approximately $10 \%$ of the cases [8].

Something to consider was the percentage of positive results in our synovial fluid/tissue cultures from preoperative joint puncture, first intraoperative and second intraoperative surgical specimens. In $85.7 \%$ of cases, identification of the etiologic pathogen from preoperative puncture was possible, in $14.3 \%$ of cases not. A negative culture is a relatively common finding in the diagnosis of PJI and is present in $0 \%-40 \%$ of cases [34] [35]. It is important to note that the presence of this microbiological result does not always mean an incorrect PJI diagnosis. This can have various reasons, such as prior use of antibiotics, which was the case in our patients who received systemic antibiotic treatment before sampling. However, many other reasons may cause a negative result, such as inadequate culture conditions for not frequent bacterial joint infections, the low presence of the etiologic pathogen, which may already have been eliminated but whose antigens, toxins, or superantigens could cause an inflammatory response, and clinical manifestations in the patient [36] [37]. In this case, new diagnostic alternatives are being investigated, such as multiplex PCR, which has been described as a reliable early diagnostic tool in patients with PJI and SIRS, where early pathogen identification is cardinal [38].

Another common possibility, though particularly in chronic PJI, of negative cultures is the presence of biofilms, where the need for a different type of culture may require procedures for accurate diagnosis, such as sonication [39] [40].

Compared to preoperative puncture, the number of positive cultures from the samples from the first surgery decreased by $10.7 \%$ from $85.7 \%$ to $75 \%$ and the number of negative cultures increased accordingly from $14.3 \%$ to $25 \%$. A possible explanation could be that after diagnosis and preoperative sampling, antibiotics were given to patients, which may have influenced other cultures even after a few hours of administration. On the other hand, the results of the cultures at the time of sampling were completely reversed at the second surgery compared to the first surgery, resulting in a $75 \%$ and $25 \%$ number of negative and positive 
cultures, respectively.

Several risk factors, including an increased ASA score, have been associated with an increased risk of infection at the surgical site. This is particularly true for patients with an ASA score $\geq 3$ [23] [41], as our results show, where the majority of our patients (80\%) had an ASA classification score of 3 (severe systemic disease) on arrival at our hospital.

Even today, PJI treatment still poses a great challenge for doctors. To make the best treatment decisions, a multidisciplinary team and an individual approach to each patient should be chosen. As reviewed [7] [8], several surgical strategies are available for the treatment of PJI. These include arthrodesis, antimicrobial suppression without surgery, amputation, open or arthroscopic debridement without removal of the prostheses or resection of the prostheses with or without reimplantation. The latter can be performed either in one step (at the time of removal) or in two steps (usually delayed by weeks). All our patients were treated with this last option, a two-step surgical strategy described as the best treatment to eradicate the infection with better patient recovery [8]. To our knowledge, there is no other study that provides a treatment strategy for patients with PJI and concurrent sepsis. Since the rapid onset of sepsis treatment is known, one of our main goals was to start the first surgery as soon as possible after admission and diagnosis of the patient. This time span was 5.45 hours on average in our study group. Although this period of time seems long at first, it is due to the fact that laboratory results have to be awaited and the surgery logistics have to be adhered to. It has been shown that a longer operation time is associated with a higher risk of infections and other complications such as heart disease, pneumonia, kidney failure and sepsis [42]. Each additional 15 - 20 minutes of surgery time is associated with a $9 \%-25 \%$ increased risk of joint infection [23] [43]. With an average of 23 minutes of surgery time in the first operation, the perioperative burden on the patient with sepsis was limited, with low blood loss.

The second planned operation took place after an average interval of 8 days and lasted 115.7 minutes on average. By this time, the patients had already improved in terms of the infection laboratory parameters and clinical symptoms, so that the long duration of the operation was associated with less risk for them. In addition, the first microbiological results were available at this point in time, which allowed for targeted antibiotic treatment.

Our results showed a high success rate of $100 \%$ in treating the infection in 10 patients who chose surgery without removal of the prostheses as a treatment option. This shows in our patient collective that the maintenance of a prosthesis in case of PJI with sepsis is justified and has a good prognosis overall. Patients who had to have their prostheses removed and re-implanted with a time delay also showed a high success rate of $100 \%$ (8 patients). These in a total follow-up period of 9.4 months on average. These confirm the validity of other studies in which the success rates of a two-stage surgical treatment were examined at $87 \%$ $100 \%$ and $72 \%$ - 95\% for hip and knee PJI [8]. 
Successful disease management could be reflected in some clinical and laboratory parameters, as our results show. Where more than half of our patients had fever at the time of admission, this was only a few percent of patients with this clinical symptom, in contrast to other studies [16] [17]. Although this symptom may not have been present in all patients and may not have been the most common in our study, if present, it could be a very specific finding for the diagnosis of PJI with sepsis [44]. Its importance is also shown by the fact that it was the first parameter to normalize and indicate successful treatment in our patients. Already 24 hours after admission, the body temperature of the patients was normal, probably due to the rapid initial surgical revision, which mainly focused on joint lavage, debridement, the use of systemic and local antibiotics and the insertion of an irrigation drainage. From the second day until discharge, the patients' body temperature remained within the normal range with no statistically significant change.

CRP is considered and inflammatory marker for many diseases and medical conditions, however, it is supportive and not definitive evidence criteria for diagnosing PJI [8], due to its lack of specificity. One must take also into consideration that in some cases, especially in patients infected with Propionibacterium acnes CRP might be in normal levels even when every other parameters suggests an infection [45]. However, the lowering of its levels strongly suggests an effective treatment as shown in our patients, in which similar to fever, its levels started to lower after the first surgery, this after 24 hours of admission. It is known that CRP half-life is known to be around 19 hours, that its concentration peak is reported to be at 48 hours [46]. However, it has been demonstrated that after total joint replacement this parameter may have a longer half-life of 62 hours [47], which might explain the reason of some peaks on the daily measurement levels of this parameter in our patients. However, a decrease pattern can clearly be seen in our results until the last day of the inpatient care. CRP release by hepatocytes is mainly controlled by cytokines release, specially IL-6 which has been proposed as a better inflammatory and follow up marker due to its concentration peak at 6 hours and a half-life of 15 hours [47] and therefore a rapid return to its normal concentrations in comparison with CRP.

As with the majority of studies, our retrospective study design is subject to certain limitations. One possible limitation was the number of patients included in the research, which must be larger in order to draw more objective conclusions. Since PJI with sepsis as a disease is rather rare, the patient population is too diverse in terms of prosthesis design, bacterial species and their resistance patterns, secondary diseases and age, it is difficult to conduct a prospective study on the same topic. The other limitation was the short follow up time.

\section{Conclusion}

In summary, the combination of acute PJI and SIRS is a complex severe clinical picture with leading symptoms such as joint pain and feeling of illness in a pro- 
dromal stage before the onset of sepsis. This period of time, in our retrospective study 5 days, should be effectively used diagnostically and therapeutically to prevent the risk of developing sepsis and thus an increased mortality. Our multi-stage surgical treatment concept of a quick and above all short first operation followed by a delayed second scheduled revision operation has proven to be successful in treating PJI in sepsis. Especially the prosthesis retention attempt seems to have a good prognosis for patients in this highly acute infection situation. The CRP and fever are important progression parameters that can indicate early successful treatment. The discrepancy in the positive microbiological culture results between the early joint puncture, the subsequent first operation and above all the blood cultures from the day of admission was striking.

\section{Conflicts of Interest}

The authors declare no conflicts of interest regarding the publication of this paper.

\section{References}

[1] Kurtz, S., Ong, K., Lau, E., Mowat, F. and Halpern, M. (2007) Projections of Primary and Revision Hip and Knee Arthroplasty in the United States from 2005 to 2030. The Journal of Bone and Joint Surgery, 89, 780-785. https://doi.org/10.2106/00004623-200704000-00012

[2] Zimmerli, W., Trampuz, A. and Ochsner, P.E. (2004) Prosthetic-Joint Infections. N The New England Journal of Medicine, 351, 1645-1654. https://doi.org/10.1056/NEJMra040181

[3] Izakovicova, P., Borens, O. and Trampuz, A. (2019) Periprosthetic Joint Infection: Current Concepts and Outlook. EFORT Open Reviews, 4, 482-494. https://doi.org/10.1302/2058-5241.4.180092

[4] Ulrich, S.D., Seyler, T.M., Bennett, D., et al. (2008) Total Hip Arthroplasties: What Are the Reasons for Revision? International Orthopaedics, 32, 597-604. https://doi.org/10.1007/s00264-007-0364-3

[5] Natsuhara, K.M., Shelton, T.J., Meehan, J.P. and Lum, Z.C. (2019) Mortality during Total Hip Periprosthetic Joint Infection. The Journal of Arthroplasty, 34, S337-S342. https://doi.org/10.1016/j.arth.2018.12.024

[6] Zachary, C., Lum, K.M.N., Shelton, T.J., Giordani, M., Pereria, G.C. and Meehan, J.P. (2018) Mortality during Total Knee Periprosthetic Joint Infection. The Journal of Arthroplasty, 33, 3783-3788. https://doi.org/10.1016/j.arth.2018.08.021

[7] Li, C., Renz, N. and Trampuz, A. (2018) Management of Periprosthetic Joint Infection. Hip \& Pelvis, 30, 138-146. https://doi.org/10.5371/hp.2018.30.3.138

[8] Tande, A.J. and Patel, R. (2014) Prosthetic Joint Infection. Clinical Microbiology Reviews, 27, 302-345. https://doi.org/10.1128/CMR.00111-13

[9] Opal, S.M. (2010) Endotoxins and Other Sepsis Triggers. Contributions to Nephrology, 167, 14-24. https://doi.org/10.1159/000315915

[10] Beesley, S.J. and Lanspa, M.J. (2015) Why We Need a New Definition of Sepsis. Annals of Translational Medicine, 3, 296.

[11] SepNet Critical Care Trials Group (2016) Incidence of Severe Sepsis and Septic Shock in German Intensive Care Units: The Prospective, Multicentre INSEP Study. 
Intensive Care Medicine, 42, 1980-1989. https://doi.org/10.1007/s00134-016-4504-3

[12] Engel, C., Brunkhorst, F.M., Bone, H.G., et al. (2007) Epidemiology of Sepsis in Germany: Results from a National Prospective Multicenter Study. Intensive Care Medicine, 33, 606-618. https://doi.org/10.1007/s00134-006-0517-7

[13] Singer, M., Deutschman, C.S., Seymour, C.W., et al. (2016) The Third International Consensus Definitions for Sepsis and Septic Shock (Sepsis-3). JAMA, 315, 801-810. https://doi.org/10.1001/jama.2016.0287

[14] Committee AHODE (2014) ASA Physical Status Classification System. American Society of Anesthesiologists.

[15] Zmistowski, B., Karam, J.A., Durinka, J.B., Casper, D.S. and Parvizi, J. (2013) Periprosthetic Joint Infection Increases the Risk of One-Year Mortality. The Journal of Bone and Joint Surgery, 95, 2177-2184. https://doi.org/10.2106/JBJS.L.00789

[16] Tseng, S.W., Chi, C.Y., Chou, C.H., et al. (2012) Eight Years Experience in Treatment of Prosthetic Joint Infections at a Teaching Hospital in Central Taiwan. Journal of Microbiology, Immunology and Infection, 45, 363-369.

https://doi.org/10.1016/j.jmii.2011.12.014

[17] Tsaras, G., Osmon, D.R., Mabry, T., Lahr, B., St. Sauveur, J., Yawn, B., Kurland, R. and Berbari, E.F. (2012) Incidence, Secular Trends, and Outcomes of Prosthetic Joint Infection: A Population-Based Study, Olmsted County, Minnesota, 1969-2007. Infection Control and Hospital Epidemiology, 33, 1207-1212. https://doi.org/10.1086/668421

[18] Gregory, D., Paul, L. and Scott, K. (1996) Aspiration of the Knee Joint before Revision Arthroplasty. Clinical Orthopedics and Related Research, 331, 132-139. https://doi.org/10.1097/00003086-199610000-00018

[19] Dale, H., Fenstad, A.M., Hallan, G., et al. (2012) Increasing Risk of Prosthetic Joint Infection after Total Hip Arthroplasty. Acta Orthopaedica, 83, 449-458. https://doi.org/10.3109/17453674.2012.733918

[20] Jamsen, E., Huhtala, H., Puolakka, T. and Teemu, M. (2009) Risk Factors for Infection after Knee Arthroplasty. A Register-Based Analysis of 43,149 Cases. The Journal of Bone and Joint Surgery, 91, 38-47. https://doi.org/10.2106/JBJS.G.01686

[21] Lenguerrand, E., Whitehouse, M.R., Beswick, A.D., Kunutsor, S.K., Burston, B., Porter, M. and Blom, A.W. (2018) Risk Factors Associated with Revision for Prosthetic Joint Infection after Hip Replacement: A Prospective Observational Cohort Study. The Lancet Infectious Diseases, 18, 1004-1014. https://doi.org/10.1016/S1473-3099(18)30345-1

[22] Inoue, D., Xu, C., Yazdi, H. And Parviza, J. (2019) Age Alone Is Not a Risk Factor for Periprosthetic Joint Infection. Journal of Hospital Infection, 103, 64-68.

[23] Namba, R.S., Inacio, M.C. and Paxton, E.W. (2013) Risk Factors Associated with Deep Surgical Site Infections after Primary Total Knee Arthroplasty: An Analysis of 56,216 Knees. The Journal of Bone and Joint Surgery, 95, 775-782. https://doi.org/10.2106/JBJS.L.00211

[24] Edwards, J.R., Peterson, K.D., Mu, Y., et al. (2009) National Healthcare Safety Network (NHSN) Report: Data Summary for 2006 through 2008, Issued December 2009. American Journal of Infection Control, 37, 783-805. https://doi.org/10.1016/j.ajic.2009.10.001

[25] Patti, J.M., Allen, B.L., Mcgavin, M.J. and Hook, M. (1994) MSCRAMM-Mediated Adherence of Microorganisms to Host Tissues. Annual Review of Microbiology, 48, 585-617. https://doi.org/10.1146/annurev.mi.48.100194.003101

[26] Foster, T.J., Geoghegan, J.A., Ganesh, V.K. and Höök, M. (2014) Adhesion, Inva- 
sion and Evasion: The Many Functions of the Surface Proteins of Staphylococcus aureus. Nature Reviews Microbiology, 12, 49-62.

https://doi.org/10.1038/nrmicro3161

[27] Patti, J.M., Bremell, T., Krajewska-Pietrasik, D., Abdelnour, A., Tarkowski, A., Ryden, C. and Höök, M. (1994) The Staphylococcus aureus Collagen Adhesin Is a Virulence Determinant in Experimental Septic Arthritis. Infection and Immunity, 62, 152-161. https://doi.org/10.1128/IAI.62.1.152-161.1994

[28] Cheung, A.L. and Fischetti, V.A. (1990) The Role of Fibrinogen in Staphylococcal Adherence to Catheters in Vitro. The Journal of Infectious Diseases, 161, 11771186. https://doi.org/10.1093/infdis/161.6.1177

[29] Lister, J.L. and Horswill, A.R. (2014) Staphylococcus aureus Biofilms: Recent Developments in Biofilm Dispersal. Frontiers in Cellular and Infection Microbiology, 4, 178. https://doi.org/10.3389/fcimb.2014.00178

[30] Parvizi, J. and Della Valle, C.J. (2010) AAOS Clinical Practice Guideline: Diagnosis and Treatment of Periprosthetic Joint Infections of the Hip and Knee. Journal of the American Academy of Orthopaedic Surgeons, 18, 771-772.

https://doi.org/10.5435/00124635-201012000-00007

[31] Parvizi, J., Gehrke, T. and Chen, A.F. (2013) Proceedings of the International Consensus on Periprosthetic Joint Infection. The Bone \& Joint Journal, 95-B, 14501452. https://doi.org/10.1016/j.arth.2017.08.034

[32] Klement, M.R., Siddiqi, A., Rock, J.M., Chen, A.F., Bolognesi, M.P. and Seyler, T.M. (2018) Positive Blood Cultures in Periprosthetic Joint Infection Decrease Rate of Treatment Success. The Journal of Arthroplasty, 33, 200-204.e1.

[33] Li, Z.L., Hou, Y.F., Zhang, B.Q., et al. (2018) Identifying Common Pathogens in Periprosthetic Joint Infection and Testing Drug-Resistance Rate for Different Antibiotics: A Prospective, Single Center Study in Beijing. Orthopaedic Surgery, 10, 235-240. https://doi.org/10.1111/os.12394

[34] Yoon, H.K., Cho, S.H., Lee, D.Y., Kang, B.-H., Lee, S.-H., Moon, D.-G., Kim, D.-H., Nam, D.-C. and Hwang. S.-C. (2017) A Review of the Literature on Culture-Negative Periprosthetic Joint Infection: Epidemiology, Diagnosis and Treatment. Knee Surgery \& Related Research, 29, 155-164. https://doi.org/10.5792/ksrr.16.034

[35] Tan, T.L., Kheir, M.M., Shohat, N., et al. (2018) Culture-Negative Periprosthetic Joint Infection: An Update in Vitro What to Expect. JBJS Open Access, 3, e0060. https://doi.org/10.2106/JBJS.OA.17.00060

[36] Ali, A., Zhu, X.F., Kwiecinski, J., et al. (2015) Antibiotic-Killed Staphylococcus aureus Induces Destructive Arthritis in Mice. Arthritis \& Rheumatology, 67, 107-116. https://doi.org/10.1002/art.38902

[37] Noorbakhsh, S, Talebi-Taher, M. and Tabatabaei, A. (2013) Identification of Bacterial Antigens and Super Antigens in Synovial Fluid of Patients with Arthritis: A Cross Sectional Study. Medical Journal of Islamic Republic of Iran, 27, 12-16.

[38] Lausmann, C., Zahar, A., Citak, M., et al. (2017) Are There Benefits in Early Diagnosis of Prosthetic Joint Infection with Multiplex Polymerase Chain Reaction? Journal of Bone and Joint Infection, 2, 175-183. https://doi.org/10.7150/jbji.22062

[39] Gbejuade, H.O., Lovering, A.M. and Webb, J.C. (2015) The Role of Microbial Biofilms in Prosthetic Joint Infections. Acta Orthopaedica, 86, 147-158. https://doi.org/10.3109/17453674.2014.966290

[40] Taha, M., Abdelbary, H., Ross, F.P., et al. (2018) New Innovations in the Treatment of PJI and Biofilms-Clinical and Preclinical Topics. Current Reviews in Musculoskeletal Medicine, 11, 380-388. https://doi.org/10.1007/s12178-018-9500-5 
[41] Namba, R.S., Inacio, M.C.S. andPaxton, E.W. (2012) Risk Factors Associated with Surgical Site Infection in 30491 Primary Total Hip Replacements. The Journal of Bone and Joint Surgery, 94-B, 1330-1338. https://doi.org/10.1302/0301-620X.94B10.29184

[42] Cheng, H., Clymer, J.W., Chen, B.P.-H., et al. (2018) Prolonged Operative Duration Is Associated with Complications: A Systematic Review and Meta-Analysis. Journal of Surgical Research, 229, 134-144. https://doi.org/10.1016/j.jss.2018.03.022

[43] Wang, Q., Goswami, K., Shohat, N., Aalirezaie, A., Manrique, J. and Parvizi, J. (2019) Longer Operative Time Results in a Higher Rate of Subsequent Periprosthetic Joint Infection in Patients Undergoing Primary Joint Arthroplasty. The Journal of Arthroplasty, 34, 947-953. https://doi.org/10.1016/j.arth.2019.01.027

[44] Shohat, N., Goswami, K., Tan, T.L., et al. (2019) Fever and Erythema Are Specific Findings in Detecting Infection Following Total Knee Arthroplasty. Journal of Bone and Joint Infection, 4, 92-98. https://doi.org/10.7150/jbji.30088

[45] Piper, K.E., Jacobson, M.J., Cofield, R.H., et al. (2009) Microbiologic Diagnosis of Prosthetic Shoulder Infection by Use of Implant Sonication. Journal of Clinical Microbiology, 47, 1878-1884. https://doi.org/10.1128/JCM.01686-08

[46] Pepys, M.B. and Hirschfield, G.M. (2003) C-Reactive Protein: A Critical Update. Journal of Clinical Investigation, 111, 1805-1812. https://doi.org/10.1172/JCI200318921

[47] Wirtz, D.C., Heller, K.-D., Miltner, O., Zilkens, K.-W. and Wolff, J.M. (2000) Interleukin-6: A Potential Inflammatory Marker after Total Joint Replacement. International Orthopaedics, 24, 194-196. https://doi.org/10.1007/s002640000136 